\title{
Comparison of micro-milling and micro-EDM operations
}

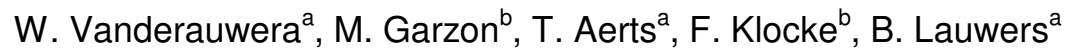 \\ ${ }^{a}$ Department of Mechanical Engineering, Katholieke Universiteit Leuven, 3000, Belgium \\ ${ }^{\mathrm{b}}$ Werkzeugmaschinenlabor WZL, Rheinisch-Westfälische Technische Hochschule Aachen, 52056, Germany
}

\begin{abstract}
The production of micro-parts often involves micro-milling and micro-EDM operations. Although both processes are competitive for certain geometries, each of both processes has its specific advantages. Driven by the current trend of hybridization of machining processes it is important to know which process or which combination of processes to use in order to attain a certain performance level. Therefore this paper compares micro-milling and micro-EDM operations specifically for the machining of rectangular shaped geometries. The comparison is based on several performance parameters like dimensional and geometrical accuracy, surface roughness, machining time and energy consumption. This initial study shows that for the considered geometries a comparable dimensional accuracy can be obtained for both processes. Besides this it is shown that micro-milling excels micro-EDM in terms of flatness of the bottom surface, surface roughness and machining time. On the contrary, micro-EDM gives better performance in terms of straightness of the walls and energy consumption. Based on these results combined machining strategies are proposed which make use of the advantages of both processes.
\end{abstract}

Keywords: micro-EDM, micro-milling, process planning

\section{Introduction}

Micro-EDM and micro-milling are frequently used techniques in the production of micro-parts like moulds (micro-injection moulding, hot embossing tools) or micro-mechanical parts. Both techniques have their specific advantages and disadvantages. Micro-EDM is a relative slow machining process but due to its contactless nature it is often used to machine very hard materials. Micro-EDM is also suitable to machine high aspect ratio holes, even with aspect ratios up to 18 [1]. On the contrary micromilling is a fast machining process able to machine complex shapes with a good surface quality. However the attainable aspect ratio is limited [2].

Nowadays more and more micro-products demand multiple machining operations [3]. Together with the current trend towards hybridisation of processes in micro-production, a proper process planning is indispensable. For this, it is essential to know how well each process performs for a certain machining case. Until now literature only reports on the micro-machining of high aspect ratio holes and ribs [4]. For other types of geometries the outcomes of these studies only serve as rough guidelines. Additionally it is important to examine, next to aspect ratio and accuracy, other relevant performance parameters in order to make a proper decision about which (combination of) processes to use.

This paper discusses the results of an initial study in which micro-milling and micro-EDM milling operations were compared. In this study the focus was on rectangular shaped cavities. The comparison between both processes is based on several performance parameters like dimensional and geometrical accuracy, roughness, machining time and energy consumption. The results of this study can then be used to define combined operations which use the advantages of both processes.

\section{Experimental plan}

\subsection{Experimental setup}

The comparison of both processes is based on machining experiments performed on a combined machining platform. The platform is based on a commercially available micro-EDM machine (Sarix SX-200) equipped with a high speed mechanical air bearing spindle (Fig. 1) which is regulated by a frequency converter to deliver rotational speeds up to $160000 \mathrm{rpm}$. The performance of both processes was examined on two types of rectangular cavities namely shallow square cavities and deep slots.

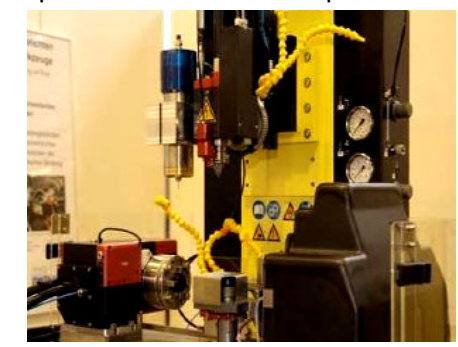

Fig. 1. Combined machining platform with micro-EDM spindle and micro-milling spindle.

For the square type geometries, cavities with three different dimensions were machined to cover 
Table 1. Dimensions and machining conditions of experimental plan.

\begin{tabular}{|c|c|c|c|c|c|c|c|c|c|c|c|c|}
\hline \multirow{3}{*}{ \# } & \multirow{3}{*}{$\begin{array}{l}\text { Length } \\
(\mathrm{mm})\end{array}$} & \multirow{3}{*}{$\begin{array}{l}\text { Width } \\
(\mathrm{mm})\end{array}$} & \multirow{3}{*}{$\begin{array}{l}\text { Depth } \\
(\mathrm{mm})\end{array}$} & \multirow{3}{*}{$\begin{array}{c}\text { Aspect } \\
\text { ratio }\end{array}$} & \multirow{3}{*}{$\begin{array}{c}\varnothing \text { Tool } \\
\text { roughing } \\
(\mathrm{mm})\end{array}$} & \multirow{3}{*}{$\begin{array}{c}\varnothing \text { Tool } \\
\text { finishing } \\
(\mathrm{mm})\end{array}$} & \multicolumn{4}{|c|}{ Micro-milling } & \multicolumn{2}{|c|}{ Micro-EDM } \\
\hline & & & & & & & \multicolumn{2}{|c|}{ Roughing } & \multicolumn{2}{|c|}{ Finishing } & \multirow{2}{*}{\begin{tabular}{|c|} 
Roughing \\
$\begin{array}{c}\mathrm{a}_{\mathrm{p}} \\
(\mathrm{mm})\end{array}$
\end{tabular}} & \multirow{2}{*}{$\begin{array}{c}\text { Finishing } \\
\begin{array}{c}\mathrm{a}_{\mathrm{p}} \\
(\mathrm{mm})\end{array}\end{array}$} \\
\hline & & & & & & & $\begin{array}{c}\mathrm{f}_{\mathrm{z}} \\
(\mathrm{mm} / \mathrm{rev})\end{array}$ & $\begin{array}{c}a_{p} \\
(\mathrm{~mm})\end{array}$ & $\begin{array}{c}\mathrm{f}_{\mathrm{z}} \\
(\mathrm{mm} / \mathrm{rev}) \\
\end{array}$ & $\begin{array}{c}a_{p} \\
(\mathrm{~mm})\end{array}$ & & \\
\hline 1 & 10 & 10 & 0.2 & 0.02 & 1 & 0.5 & 0.04 & 0.04 & 0.03 & 0.005 & 0.0085 & 0.0009 \\
\hline 2 & 1 & 1 & 0.2 & 0.2 & 0.3 & 0.2 & 0.01 & 0.01 & 0.01 & 0.0015 & 0.0015 & 0.0015 \\
\hline 3 & 0.3 & 0.3 & 0.1 & 0.33 & 0.1 & 0.1 & 0.0028 & 0.0015 & 0.0018 & 0.0015 & 0.0005 & 0.0005 \\
\hline 4 & 2.5 & 0.55 & 2.5 & 4.5 & 0.5 & & 0.03 & 0.005 & & & 0.0035 & \\
\hline 5 & 2.5 & 0.55 & 4 & 7.3 & 0.5 & & 0.01 & 0.005 & & & 0.0035 & \\
\hline
\end{tabular}

the whole range from meso- to micro-geometries (10 $-10^{-3} \mathrm{~mm}$, see Table 1 case \#1-3). The machining consisted of a roughing operation with mostly a large tool followed by a finishing operation of walls and floors with a smaller tool. For the milling operations it is chosen to machine at the maximum allowable feed per tooth $\left(f_{z}\right)$ and maximum allowable axial depth of cut $\left(a_{p}\right)$. Because the feedrate of the machining platform is limited to $20 \mathrm{~mm} / \mathrm{s}$ a fixed rotational speed (30400rpm) has been chosen so that for none of the milling tools the feedrate would exceed the maximum feedrate of the machine. Also the effect of the machining strategy was examined by performing concentric-out and zig-zag movements.

For the slot type cavities only the cavity depth was changed in order to investigate the performance of both processes as a function of the aspect ratio (up to 7.3 , see Table 1 case \#4-5). Unlike the square cavities, only a roughing operation was performed. For each slot a milling tool was chosen which maximum allowable cutting depth equals the depth of the slot. The milling operation of the slots was performed at maximum $\mathrm{f}_{\mathrm{z}}$ and maximum $\mathrm{a}_{\mathrm{p}}$. One rotational speed (40000rpm) was chosen so that none of the resulting feedrates would exceed the maximum feedrate of the machine.

The experiments were performed with the same $\mathrm{NC}$-codes for both processes except for the $\mathrm{a}_{\mathrm{p}}$ values. Hardened steel nr. 1.2343 (DIN X38CrMoV5-1) with a hardness of $57 \mathrm{HRC}$ was used as workpiece material. As process fluid lonoGrind63 was chosen which has properties to act as well as a cutting fluid as a dielectric. The electrodes are tungsten carbide rods whereas the milling tools are coated solid carbide end mills with two flutes (Table 1). Two replications were performed for each case.

\subsection{Measurement setup}

The comparison of both processes is based on several performance parameters like dimensional accuracy (length, width, depth), geometrical accuracy (inclination angle of side walls, flatness of bottom surface), surface roughness, machining time and energy consumption. Three profile meters were used: Perthometer PGK 120-Mahr for the surface roughness measurements, Taylor Hobson Form Talysurf $120 \mathrm{~L}$ for the flatness measurements and Mahr MarSurf XC2 for the depth measurements. The length, width and inclination angle of the side walls were quantified with a digital microscope, type Keyence VHX-100. The energy measurements were done with a Chauvin-Arnoux CA8335 power analyser.

\section{Results \& discussion}

\subsection{Dimensional accuracy}

When looking at the length accuracy for the square cavities, the experiments showed that no significant distinction could be made for the examined machining strategies. Fig. 2 gives an overview of the deviations from the desired values for the examined cases for both processes. This figure shows that only for the smallest square cavities (case \#3) micro-EDM gives a significant higher length accuracy (significance level $99 \%$ or $\alpha=0.01$ ). For the slots a distinction should be made between the length and width accuracy. The width accuracy is comparable for both processes. On the contrary, the length accuracy seems to differ for the deepest slots (case \#5) although the significance level for this is only $50 \%$. The results given here should be carefully treated. The results shown in Fig. 2 are measured with one microscope by one person. When the same dimensions are measured with the same microscope but with another magnitude a difference of up to $15 \mu \mathrm{m}$ between the measurements was noticed. Moreover the inter-person deviation can run up to $45 \mu \mathrm{m}$ whereas the inter-microscope deviations run up to $38 \mu \mathrm{m}$. It can be concluded that this measurement method is not reliable enough for these type of cavities to state hard conclusions.

Opposed to the length/width measurements, the depth was measured tactile. Fig. 3 shows the results of these measurements. No significant difference in depth accuracy is noticed between the two processes for the square cavities. On the other hand, in case of slots (case \#4-5) micro-milling results in a significant higher depth accuracy $(\alpha=0.01$ for slots $\# 4 ; \alpha=0.1$ for slots \#5). This can be explained by the fact that the depth accuracy of the micro-EDM operations is highly dependent on the accuracy of the wear compensation. For large depths, like these slots, the effect of a suboptimal wear compensation leads to a larger deviation from the desired depth.

\subsection{Geometrical accuracy}

The geometrical accuracy of the considered cavities is first assessed by examining the straightness of the edges. No significant distinction could be made between the different machining strategies and between both processes. However, some milled edges showed locally some distortions. These are probably caused by tool deflections and vibrations due to accelerations on the cavity corners. 


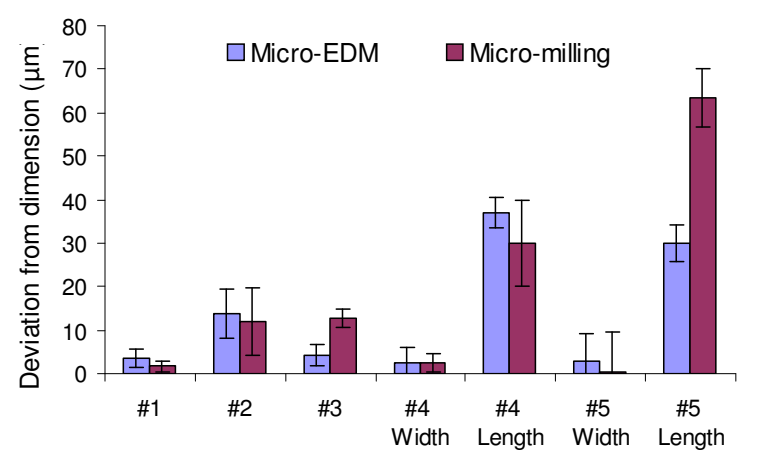

Fig. 2. Comparison of length and width accuracy for micro-milling and micro-EDM.
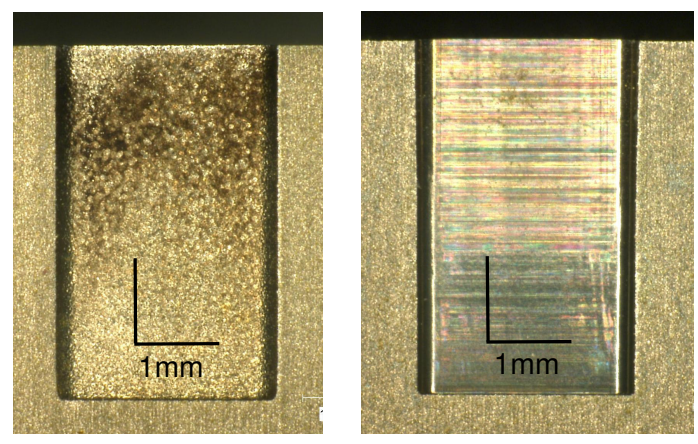

Fig. 4. Section view of slots case \#5 (left: micro-EDM; right: micro-milling).

An important performance parameter for the slot type cavities is the inclination angle of the side walls. Optical measurements showed that both for micromilling as for micro-EDM the inclination angle approximates $90^{\circ}$ (Fig. 4). Only deviations up to $1^{\circ}$ were measured.

Another important performance parameter is the flatness. For the square cavities (only case \#1 was large enough to measure) the flatness of the bottom surface has been examined whereas for the slots the flatness of the walls (after cutting the slot in two) was examined. An examination of the bottom surfaces showed that there is a significant difference between both machining processes $(\alpha=0.01)$. Generally micromilling operations lead to a better bottom surface flatness (Fig. 5). When looking at the surface profile it can be noticed that there is a strong dependence on the machining strategy. Moreover, in case of a concentric-out strategy the surface profile is dependent on the machining process. In case of micro-milling the largest deviations exist on the diagonals of the bottom surface. These are points on the tool path were the milling tool needs to slow down and accelerate again. Hence more time is spent at these points and it can be assumed that slightly more material is removed at these locations. In case of micro-EDM operations the obtained bottom surface profile is strongly influenced by the wear compensation strategy. Fig. 5 shows that

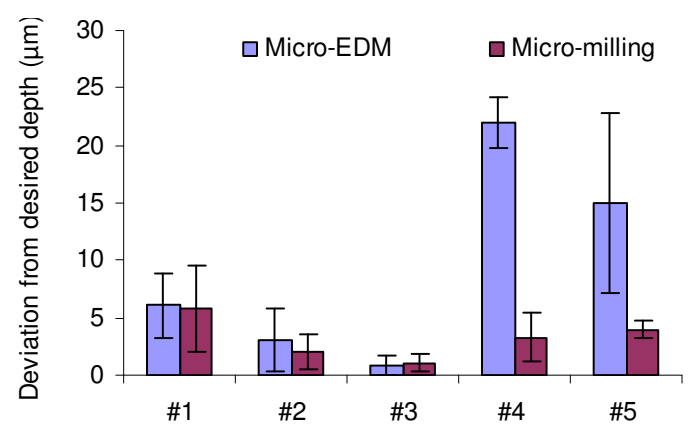

Fig.3. Comparison of depth accuracy for micromilling and micro-EDM.

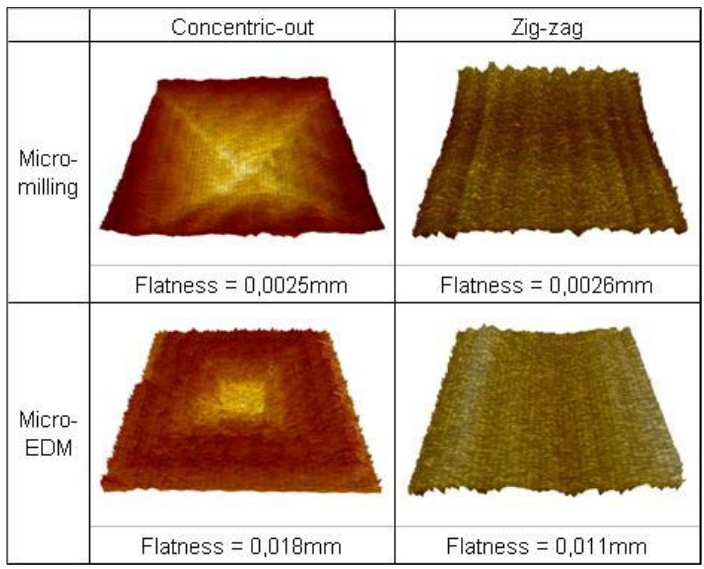

Fig. 5. Comparison of bottom surface profiles for micro-EDM and micro-milling.

in case of concentric-out movements the bottom surface has a pyramidal shape. The wear compensation does not compensate enough (increase of depth from center to boundaries) for the higher than expected wear. In case of a zig-zag movement for micro-EDM operations, a better flatness is obtained because the electrode is moving constantly from one side of the cavity to the other side. In case of a bad wear compensation strategy the result will be an inclined bottom surface but the deviations from this inclined surface will be smaller than those noticed with a concentric-out movement. For the slot type cavities the flatness of the side walls is independent of the cavity depth and comparable for both operations.

\subsection{Surface roughness}

The surface roughness of the bottom surface (square cavities) as well as the surface roughness of the side walls (slots) was compared for both processes (Table 2). For the square cavities only the surface roughness of case \#1 could be measured. When comparing the results for these cavities it was noticed that the machining strategy does not influence the roughness. However, the measurements showed that in case of micro-milling a significant better roughness can be obtained $(\alpha=0.001)$. The roughness of the side walls was measured for the slot type cavities. No 
Table 2. Bottom and side wall surface roughness for micro-milling and micro-EDM.

\begin{tabular}{|c|c|c|c|c|}
\hline \multirow{2}{*}{} & \multicolumn{2}{|c|}{ Micro-milling } & \multicolumn{2}{c|}{ Micro-EDM } \\
\cline { 2 - 5 } & $R a(\mu \mathrm{m})$ & $\mathrm{Rz}(\mu \mathrm{m})$ & $\mathrm{Ra}(\mu \mathrm{m})$ & $\mathrm{Rz}(\mu \mathrm{m})$ \\
\hline $\begin{array}{c}\text { Bottom } \\
\text { surface }\end{array}$ & 0.09 & 0.64 & 0.59 & 4.57 \\
\hline $\begin{array}{c}\text { Side } \\
\text { walls }\end{array}$ & 0.02 & 0.17 & 0.58 & 3.19 \\
\hline
\end{tabular}

Table 3. Comparison of machining time, material removal rate and energy consumption for micro-milling and micro-EDM.

\begin{tabular}{|c|c|c|c|c|c|c|}
\hline \multirow{2}{*}{$\#$} & \multicolumn{3}{|c|}{ Micro-EDM } & \multicolumn{3}{c|}{ Micro-milling } \\
\cline { 2 - 7 } & $\begin{array}{c}\text { ime }^{*} \\
(\mathrm{~min})\end{array}$ & $\begin{array}{c}\text { MRR } \\
\left(\mathrm{mm}^{3} / \mathrm{m} \text { in }\right)\end{array}$ & $\begin{array}{c}\text { Energy } \\
\text { consumption } \\
(\mathrm{kWh})\end{array}$ & $\begin{array}{c}\text { Time } \\
(\mathrm{min})\end{array}$ & $\begin{array}{c}\text { MRR } \\
\left(\mathrm{m} \mathrm{m}^{3} / \mathrm{min}\right)\end{array}$ & $\begin{array}{c}\text { Energy } \\
\text { consumption } \\
(\mathrm{kWh})\end{array}$ \\
\hline 1 & 305 & 0.066 & 0.254 & 21 & 1.01 & 0.061 \\
\hline 2 & 47 & 0.004 & 0.04 & 10 & 0.02 & 0.029 \\
\hline 3 & 15 & 0.001 & 0.013 & 6 & 0.002 & 0.017 \\
\hline 4 & 48 & 0.072 & 0.04 & 10 & 0.325 & 0.035 \\
\hline 5 & 91 & 0.06 & 0.076 & 25 & 0.221 & 0.085 \\
\hline
\end{tabular}

* without WEDG-time

significant difference was noticed in relation to the cavity depth. Here again micro-milling results in a significant lower surface roughness $(\alpha=0.001)$.

\subsection{Machining time and energy consumption}

For all machining operations in an industrial environment machining time and material removal rate (MRR) are important performance parameters. Table 3 lists the machining times and MRR of the tested cases. It can be seen that micro-milling outperforms microEDM for all the tested cases, even for the smallest cavities (case \#3) for which the rotational speed and hence the feedrate could have been higher. For both micro-milling as micro-EDM the finishing operations (wall and floor) accounted for the largest part of the total machining time.

The total energy consumption of the considered machining platform can be attributed to four groups of energy consumers: fluid conditioning system, machine controller, EDM generator and spindle unit. For the same geometry and tool movement the energy consumption between both processes differs only in the energy consumption of the EDM generator and spindle unit. The energy consumption of the EDM generator is more or less constant (ca. 50W) whereas the energy consumption of the spindle is dependent on the rotational speed. When looking at the total energy consumption of the operation (Table 3 ) it can be noticed that in some cases micro-EDM consumes less energy compared to micro-milling even though the micro-EDM machining time is much higher. In order to minimise the energy consumption a combined operation could be applied in which roughing is done with a fast micro-milling operation followed by a less energy consuming micro-EDM finishing operation.

\section{Conclusions}

This paper described the results of an initial study which compared micro-EDM milling and micro-milling for the machining of rectangular shaped cavities, this for several performance parameters. When making an assessment about the accuracy of both processes it is important to have a reliable measurement technique. The poor reliability of the optical measurements made it impossible to make hard conclusions about the dimensional accuracy. In order to correctly assess the dimensional accuracy a more reliable method is needed.

This study showed that in case of micro-EDM operations the depth accuracy and flatness are strongly dependent on the performance of the wear compensation strategy, mostly resulting in a lower accuracy compared to micro-milling. On the other hand, the quality of the cavity edges is better in case of micro-EDM probably due to the higher accelerations on curves by micro-milling. It was also shown that micromilling results in a lower surface roughness than microEDM. Although these differences were noticed, both processes are competitive for machining the considered aspect ratios. When machining higher aspect ratio cavities micro-EDM becomes more favourable. Micro-milling can then act as a roughing operation until the allowable milling depth of the tool, followed by a micro-EDM operation without the loss of accuracy.

Finally this study pointed out that although micromilling is faster than micro-EDM the energy consumption of the considered machining platform can be higher for micro-milling.

\section{Acknowledgements}

This research has been carried out within the EUFP7-NMP project Integ- $\mu$ (NMP-2007-3.5-2).

\section{References}

[1] Yu Z.Y., Rajurkar K.P., Shen H. High Aspect Ratio and Complex Shaped Blind Micro Holes by Micro EDM. CIRP annals, Vol. 51/1, 2002, pp 359-362.

[2] Friedrich C. and Vasile M. Development of the Micromilling Process for High-Aspect-Ratio Microstructures. Journal of microelectromechanical systems, Vol. 5/1, 1996, pp 33-38.

[3] Schubert A., Groß S., Schulz B., Eckert U. Sequential combination of micro-milling and laser structuring for manufacturing of complex microfluidic structures. Proceedings LiM, 2011, pp 221229.

[4] Uriarte L., Ivanov A., Oosterling H., Staemmler L., Tang P.T., Allen D. A Comparison between Microfabrication Technologies for Metal Tooling. Proceedings of the Institution of Mechanical Engineers, Part C: Journal of Mechanical Engineering Science 220, 2005, pp 1665-1676. 